Research Paper

\title{
Numb Protects Renal Proximal Tubular Cells from Puromycin Aminonu- cleoside-Induced Apoptosis through Inhibiting Notch Signaling Pathway
}

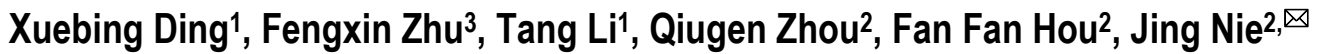 \\ 1. Department of Nephrology, The First Affiliated Hospital of Sun Yat-sen University, Guangzhou, China \\ 2. Devision of Nephrology, Nanfang Hospital, Southern Medical University, Guangdong Provincial Institute of Nephro- \\ logy, Guangzhou, China \\ 3. Department of Nephrology, Huangpu Division of The First Affiliated Hospital of Sun Yat-sen University, Guangzhou, \\ China
}

$\triangle$ Corresponding author: Jing Nie, MD, Ph.D. Division of Nephrology, Nanfang Hospital, Southern Medical University, Guangdong Provincial Institute of Nephrology, 1838 North Guangzhou Ave, Guangzhou 510515 Tel: 86-20-62787972 Fax: 86-20-83644607 E-Mail: niejing@fimmu.com

(C) Ivyspring International Publisher. This is an open-access article distributed under the terms of the Creative Commons License (http://creativecommons.org/ licenses/by-nc-nd/3.0/). Reproduction is permitted for personal, noncommercial use, provided that the article is in whole, unmodified, and properly cited.

Received: 2011.01.24; Accepted: 2011.03.11; Published: 2011.03.16

\begin{abstract}
Numb was originally discovered as an intrinsic cell fate determinant in Drosophila by antagonizing Notch signaling. The present study is to characterize the role of Numb in oxidative stress-induced apoptosis of renal proximal tubular cells. Exposure of NRK52E cells to puromycin aminonucleoside (PA) resulted in caspase 3-dependent apoptosis. Numb expression was downregulated by PA in a time- and dose-dependent manner. Knocking down endogenous Numb by siRNA sensitized NRK52E cells to PA-induced apoptosis, whereas overexpressing Numb protected NRK52E cells from PA-induced apoptosis. Moreover, PA activated Notch signaling in a time- and dose-dependent manner as indicated by increased expression of the intracellular domain of Notch and Hes-I. Notch signaling inhibitor DAPT significantly attenuated Numb siRNA-augmented apoptosis. On the other hand, overexpression of intracellular domain of Notch I could reverse the protective effect of Numb on PA-induced apoptosis. Taken together, our data demonstrated that, in renal proximal tubular cells, Numb functions as a protective molecule on PA-induced apoptosis through antagonizing Notch signaling activity.
\end{abstract}

Key words: Numb, apoptosis, renal proximal tubular cells, Notch

\section{Introduction}

Acute kidney injury (AKI) is a common renal disease with a prevalence of 10 to $30 \%$ in patients in critical care units. Despite technological advances in renal replacement therapy, the high mortality of patients with AKI has not changed in the past decades and remains $>50 \%$ [1]. AKI induces injury in the cortical proximal tubules and a more severe, generally lethal injury in the outer medullary proximal tubules [2]. Apoptotic cell death has been documented in humans and in experimental animal models of AKI.
Inhibition of apoptotic cell death is shown to ameliorate the injury and inflammation $[2,3]$. Increased expression of lethal cytokines and receptors (FasL, Fas) $[4,5]$, aberrant expression of apoptotic regulators (Bcl2, Bcl-xL, Bax) [4, 6], and caspase activation have been implicated in apoptotic cell death in AKI [7]. Recent studies demonstrated that the generation of reactive oxygen species (ROS) plays an important role in this process $[8,9]$. These free radicals can attack a wide variety of cellular components including DNA, 
and proteins and thereby enhancing the destruction of the cell structure and loss of cell function [10]. ROS also increase the production of proinflammatory cytokines that can further accelerate apoptotic signaling and cellular damage [11].

Numb was originally discovered as an intrinsic cell fate determinant in Drosophila, whereby asymmetric segregation of Numb during mitosis results in daughter cells acquiring different fates [12]. Genetic evidence in Drosophila indicates that Numb influence cell fate by negatively regulating the Notch signaling pathway [13-15]. Numb contains two protein-protein interaction domains: a phosphotyrosine- binding (PTB) domain and a proline-rich region (PRR), the latter of which functions as an SH3-binding domain $[15,16]$. Whereas only one form of Numb has been identified in Drosophila, mammals produce four isoforms of Numb that differ in the length of their PTB (lacking or containing an 11 amino acid insert) and PRR (lacking or containing a 48 amino acid insert) domains $[17,18]$. It has been demonstrated that, in PC12 cells, short PTB domain Numb isoforms sensitize cells to Nerve Growth Factor deprivation-induced apoptosis involves elevations in oxyradical production [19]. In breast cancer, Numb behaves as a tumor suppressor by preventing p53 degradation [20]. Taken together, these studies suggest a role of Numb on apoptosis. The aim of the present study was to examine the role of Numb in the regulation of renal proximal tubular cell (RPTC) apoptosis induced by oxidative stress and the underlying pathway.

\section{Materials and Methods}

\section{Reagents and Antibodies}

Normal rat kidney epithelial (NRK52E) cells were purchased from American Type Culture Collection (Manassas, VA); Dulbecco's modified Eagle's medium (DMEM), fetal bovine serum (FBS) and trypsin/EDTA solution were purchased from Invitrogen (Carlsbad, CA); Antibodies against Numb and cleaved caspase-3 were purchased from Cell Signaling (Danvers, MA); Antibodies against Notch-1, Hes-1 and p53 were purchased from Santa Cruz Biotechnology Inc (Santa Cruz, CA). $\gamma$-secretase inhibitor DAPT was purchased from Calbiochem (San Diego, CA). GAPDH antibody, Annexin V-FITC Apoptosis Detection Kit and puromycin aminonucleoside were from Sigma-Aldrich (St. Louis, MO).

\section{Plasmid and Virus construct}

pCDNA-NICD plasmid was a gift from Dr. Jane McGlade (University of Toronto, Canada). E1 and E3 regions deficient serotype 5 recombinant adenovirus vector that tagged with hemagglutinin sequence and contained a cytomegalovirus (CMV) promoter driving mouse cDNA fragment encoding Numb (pAd-HA-Numb) was constructed and generated by SinoGenoMax Co. Ltd (www.sinogenomax.com/en/, Beijing, China). The Ad-CMV-HA was used as control.

\section{siRNA Transfection}

Oligonucleotide siRNA duplex was synthesized by Shanghai Gene Pharma (Shanghai, China). The sequence of Numb siRNA was: 5'-GGACCTCATAGTTGA CCAG-3'. The transfection of siRNA in NRK52E cells was carried out with Lipofectamine 2000 (Invitrogen) according to the manufacturer's instruction.

\section{Cell culture and treatment}

NRK52E cells were grown in DMEM medium supplemented with $10 \% \mathrm{FBS}$ at $37^{\circ} \mathrm{C}$ in a humidified $5 \% \mathrm{CO}_{2}$ incubator. To overexpress Numb, 70\% confluent NRK52E cells were infected with adenovirus at a multiplicity of infection (MOI) of 100 viral particles per cell in DMEM medium supplemented with 2\% FBS and allowed to grow $24 \mathrm{~h}$ at $37^{\circ} \mathrm{C}$ in a humidified $5 \% \mathrm{CO}_{2}$ incubator.

To examine the effect of $\gamma$-secretase inhibitor DAPT on PA induced apoptosis, NRK52E cells infected with pAd-HA-Numb were pre-incubated with $10 \mu \mathrm{mol} / \mathrm{L}$ DAPT for 15 minutes before PA treatment. The same amount of DMSO was used as vehicle control.

\section{Flow cytometric analysis}

Cells under various experimental conditions were first harvested with EDTA-free trypsin, and washed twice with PBS. After centrifugation, cells were double-stained with Annexin V-FITC and PI using Annexin V-FITC Apoptosis Detection Kit according to the manufacturer's instruction and analyzed by Cytomics FC 500 Flow Cytometer (Beckman Coulter, Fullerton, CA) and CellQuestTM (BD Biosciences) software. Cells positive for Annexin V but negative for PI were considered to be apoptotic cells.

\section{Western Blot Analysis}

Harvested cells were homogenized in lysis buffer $(50 \mathrm{mmol} / \mathrm{L}$ Hepes pH 7.5, $150 \mathrm{mmol} / \mathrm{L} \mathrm{NaCl}, 10 \%$ glycerol, $1 \%$ Triton X-100, $1.5 \mathrm{mmol} / \mathrm{L} \mathrm{MgCl}_{2}, 1$ $\mathrm{mmol} / \mathrm{L}$ EGTA, $10 \mathrm{mmol} / \mathrm{L} \mathrm{NaF}, 10 \mathrm{mmol} / \mathrm{L}$ $\mathrm{Na}_{4} \mathrm{P}_{2} \mathrm{O}_{7}, 1 \mathrm{mmol} / \mathrm{L} \mathrm{Na} \mathrm{VO}_{5}, 1 \mathrm{mmol} / \mathrm{L}$ phenylmethylsulfonyl fluoride, $10 \mu \mathrm{g} / \mathrm{ml}$ leupeptin and $20 \mu \mathrm{g} / \mathrm{ml}$ aprotinin) with the protease inhibitor complete (Roche, Mannheim, Germany). Protein was quantified by the Bradford assay (Bio-Rad, Hercules, CA), equal amount of protein were separated on 
SDS-polyacrylamide gels, and transferred onto nitrocellulose membranes (Amersham Biosciences, Piscataway, NJ). After blocking in 5\% skim milk for $1 \mathrm{~h}$ at room temperature, membranes were incubated with indicated primary antibody at $4^{\circ} \mathrm{C}$ overnight followed by horseradish peroxidaseconjugated second antibody and detected by chemiluminescence (Amersham Biosciences, Piscataway, NJ). Quantification of the Western blot data were performed by measuring the intensity of the hybridization signals using the Image analysis program (Fluor-ChemTM 8900, Alpha Inotech).

\section{Statistical Analyses}

The data are expressed as means \pm SD. Statistical analysis was performed with standard statistical software (SPSS for Windows, version 13.0). Comparisons between two groups were performed using the Student's $t$ test while comparisons involving more than two groups were performed by one-way ANOVA. A $\mathrm{P}<0.05$ was considered as statistically significant.

\section{Results}

\section{PA induced RPTC apoptosis in a caspa- se-3-dependent manner}

To understand the mechanism of RPTC apoptosis induced by oxidative stress, we treated NRK52E cells with various amount of PA for $24 \mathrm{~h}$. FACS was performed to measure apoptosis and Annexin $\mathrm{V}^{+} \mathrm{PI}^{-}$ cells were detected as apoptotic cells. PA induced RPTC apoptosis in a dose-dependent manner. As shown in Figure 1A, there was 8-fold increase in apoptotic cell number after exposure to $150 \mu \mathrm{g} / \mathrm{ml}$ of PA for $24 \mathrm{~h}$ compared with control cells $(1.8 \%$ of control cells were detected as apoptotic cells, whereas, $4.13 \%, 10.2 \%$ and $15.2 \%$ of cells were detected as apoptotic cells after $50 \mu \mathrm{g} / \mathrm{ml}, 100 \mu \mathrm{g} / \mathrm{ml}$ and $150 \mu \mathrm{g} / \mathrm{ml}$ PA treatment, respectively).

Caspase- 3 is an active cell death protease involved in both the extrinsic and intrinsic pathway of apoptosis. To determine whether PA activates caspase-3, we examined the protein level of cleaved caspase-3 in the NRK52E cells after PA treatment. As shown in Figure 1B-C, the level of cleaved caspase- 3 was significant increased after $50 \mu \mathrm{g} / \mathrm{ml}$ PA treatment compared to control cells.
A

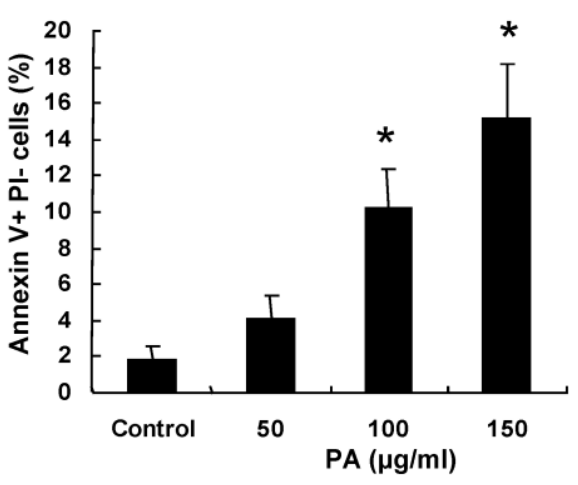

B

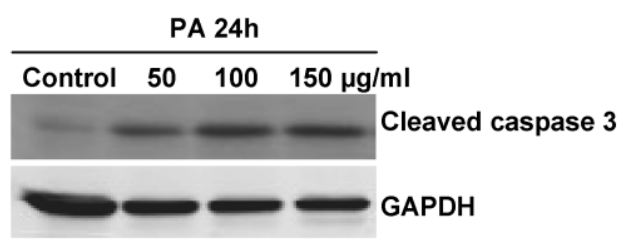

C

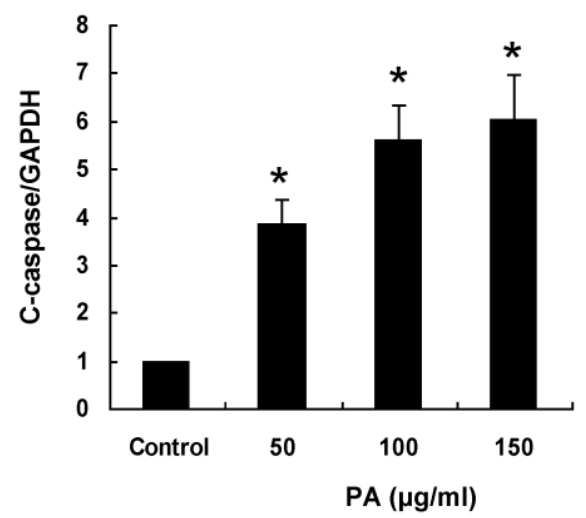

Figure I. PA induces RPTC apoptosis in a caspase-3 dependent manner. NRK52E cells were treated with indicated amount of PA for $24 \mathrm{~h}$, and apoptosis was analyzed as described in the Methods section. (A) Quantitative analysis of cell apoptosis by flow cytometry. The percentage of apoptotic cells was identified with Annexin $\mathrm{V}^{+} \mathrm{PI}$. (B) Western blot analysis of cleaved caspase- 3 expression in response to PA treatment. (C) Graphic representation of relative abundance of cleaved caspase-3 normalized to GAPDH. Each graph represents results from at least three independent experiments, and each data represents mean \pm SD from three independent experiments. $* P<0.05$ versus control without PA treatment. 


\section{PA downregulated Numb expression in a time- and dose-dependent manner}

In order to explore the role of $\mathrm{Numb}$ in PA-induced RPTC apoptosis, we first examined the expression of Numb in response to PA treatment. As shown in Figure 2A-B, Numb expression began to decrease $12 \mathrm{~h}$ after PA treatment and almost completely eliminated $24 \mathrm{~h}$ after the treatment. To further investigate whether PA-induced Numb downregulation in NRK52E cells was dose-dependent, we treated NRK52E cells with incremental concentrations of PA from 50 to $150 \mu \mathrm{g} / \mathrm{ml}$ for $24 \mathrm{~h}$. Western blot analysis revealed that the protein level of Numb decreased progressively with increased concentrations of PA. At

A

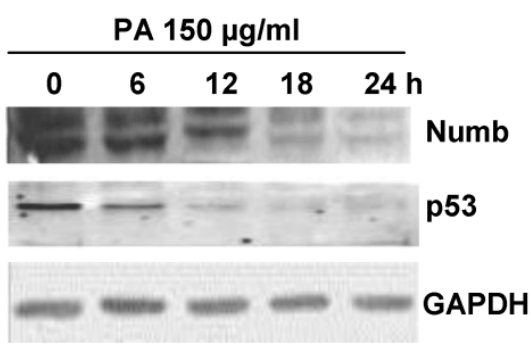

C

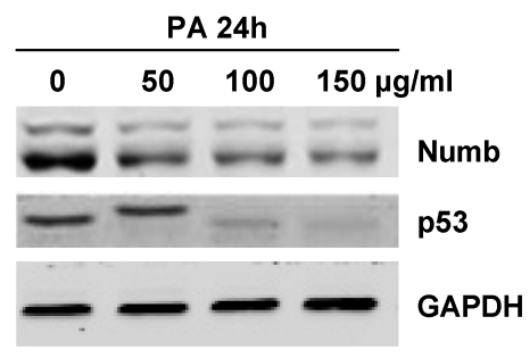

$100 \mu \mathrm{g} / \mathrm{ml}$ of PA, Numb was decreased to approximately $40 \%$ of that in control cells (Figure 2C-D).

Previous study has demonstrated that Numb could inhibit p53 degradation and thus maintains normal apoptotic cycles of mammary epithelial cells [20]. We therefore examined p53 protein level in NRK52E cells after PA treatment. PA treatment resulted in a time- and dose-dependent downregulation of p53 expression, which is associated with the downregulation of Numb (Figure 2). Taken together, the cumulative data in both figure 1 and 2 indicated that PA-induced RPTC apoptosis was independent of p53 pathway.

B

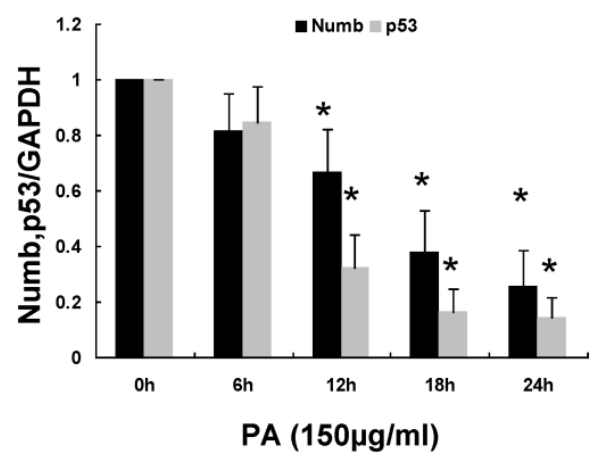

D

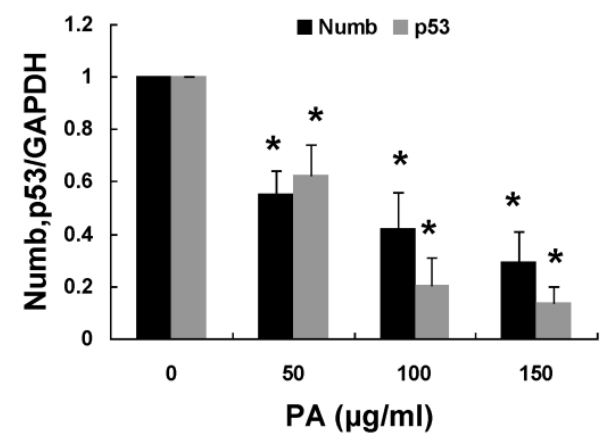

Figure 2. PA decreased Numb and $p 53$ expression in NRK52E cells in a time- and dose-dependent manner. (A) NRK52E cells were treated with $150 \mu \mathrm{g} / \mathrm{ml}$ of PA for the indicated time period. Numb and $\mathrm{p} 53$ protein level was analyzed by Western blot. GAPDH was used to verify equivalent loading. (B) Graphic representation of relative abundance of Numb or p53 normalized to GAPDH. (C) NRK52E cells were treated with indicated amount of PA for $24 \mathrm{~h}$. Numb and p53 protein level was analyzed by Western blot. GAPDH was used to verify equivalent loading. (D) Graphic representation of relative abundance of Numb or p53 normalized to GAPDH. Each graph represents results from at least three independent experiments, Data are given as means \pm SD from three independent experiments. $* P<0.05$ versus control without PA treatment. 


\section{Numb protects NRK52E cells from PA-induced apoptosis}

To determine whether PA-induced downregulation of Numb is involved in the induction of apoptosis, we knocked down endogenous Numb using siRNA prior to administrating PA. Downregulation of endogenous Numb by siRNA was demonstrated by western blot (Figure 3A). FACS showed that exposure of scramble siRNA transfected cells to $100 \mu \mathrm{g} / \mathrm{ml}$ of PA resulted in a 3-fold increase in apoptosis compared to that of scramble siRNA transfected cells without PA treatment (Figure 3D).

A

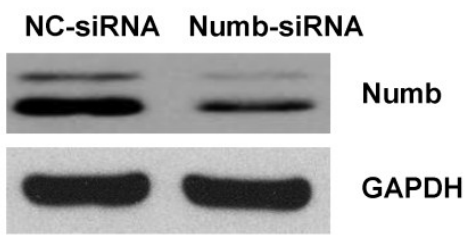

B

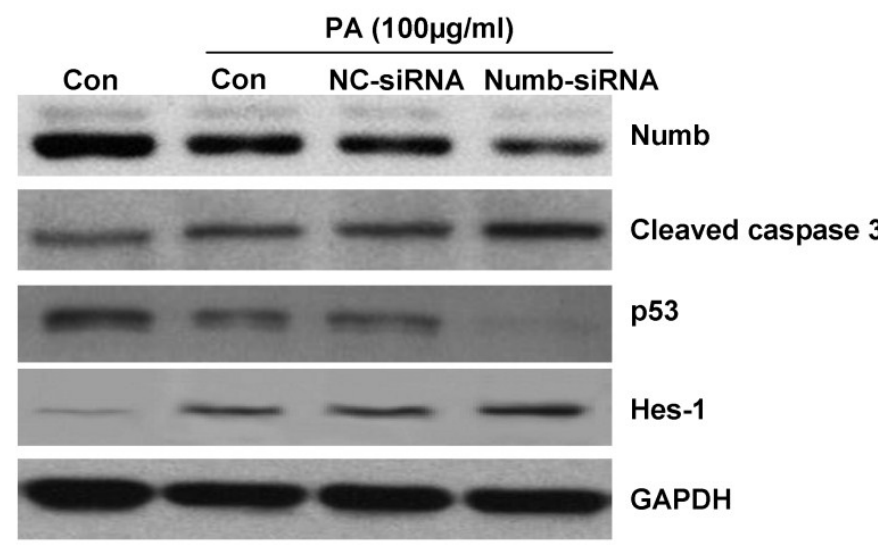

C
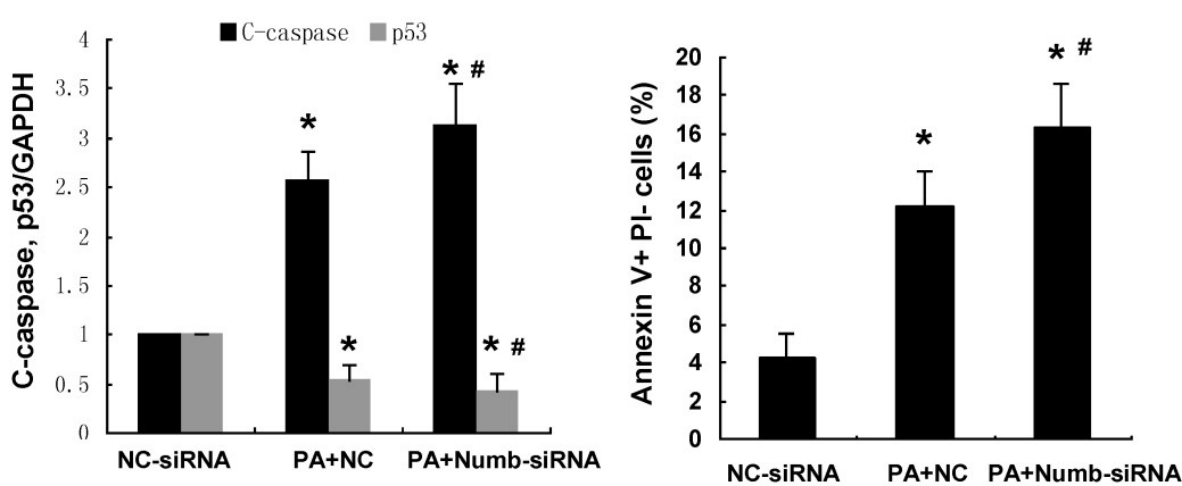

Figure 3. Knockdown endogenous Numb expression by siRNA promotes PA-induced NRK52E apoptosis. (A) The decreased protein level of Numb by siRNA was demonstrated by Western blot. (B) NRK52E cells were transfected with Numb siRNA or scramble siRNA $24 \mathrm{~h}$ before PA treatment ( $100 \mu \mathrm{g} / \mathrm{ml}$ for $24 \mathrm{~h})$. Western blot was performed to analyze the expression of Numb, p53, Hes-I and cleaved caspase-3. GAPDH was used to verify equivalent loading. (C) Graphic representation of relative abundance of $\mathrm{p} 53$ and cleaved caspase- 3 normalized to GAPDH. Each graph represents results from at least three independent experiments, and each data represents mean \pm SD from three independent experiments. (D) Quantitative analysis of cell apoptosis by flow cytometry. The percentage of apoptotic cells was identified with Annexin $\mathrm{V}^{+} \mathrm{Pl}^{-}$. Data are given as mean \pm SD from three independent experiments, $* P<0.05$ versus scramble siRNA transfected cells. ${ }^{\#} P<0.05$ versus $P A$ treated scramble siRNA transfected cells. 
Numb siRNA transfection conferred a further $30 \%$ increase in PA-induced apoptosis compared to scramble siRNA transfected cells (Figure 3D). A significant increase of cleaved caspase- 3 by Numb-siRNA was detected by Western blot (Figure 3B, 3C). In addition, Western blot analysis revealed that the protein level of p53 was reduced in Numb siRNA transfected cells compared with scramble siRNA transfected cells (Figure 3B, 3C). The expression of Hes-1, the downstream target gene of Notch signaling, was increased in Numb siRNA transfected cells.
We further confirmed the effect of Numb on PA-induced cell apoptosis by infecting NRK52E cells with adenovirus encoding HA-tagged Numb (pAd-HA-Numb). As shown in Figure 4, Numb overexpression provided marked protection from PA-induced caspase- 3 activation and cell apoptosis. Consistent with the result of Figure 3, Numb overexpression led to a remarkable increase of p53 protein expression and a dramatic decrease of Hes-1 expression compared to control cells (Figure 4A). Taken together, these data demonstrated that Numb functions as a survival molecule in PA-induced RPTC apoptosis.

A

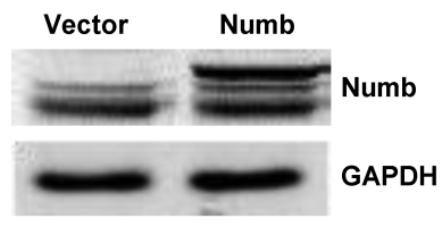

B

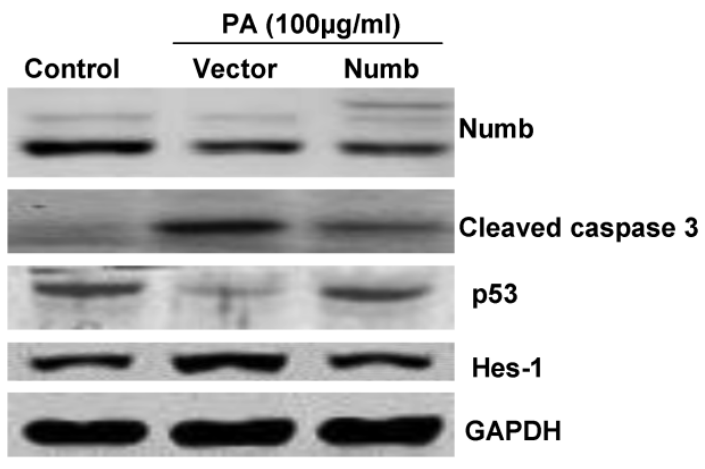

C

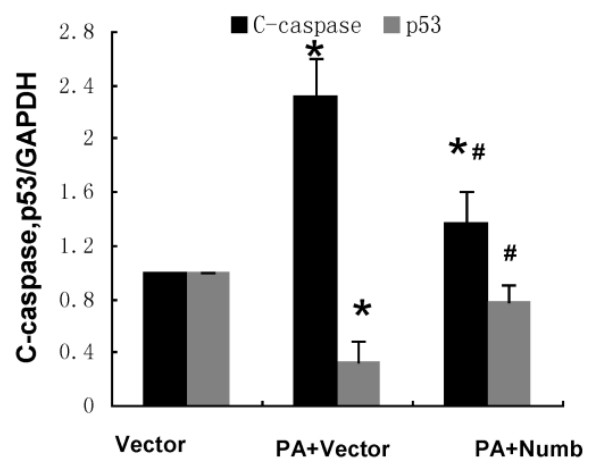

D

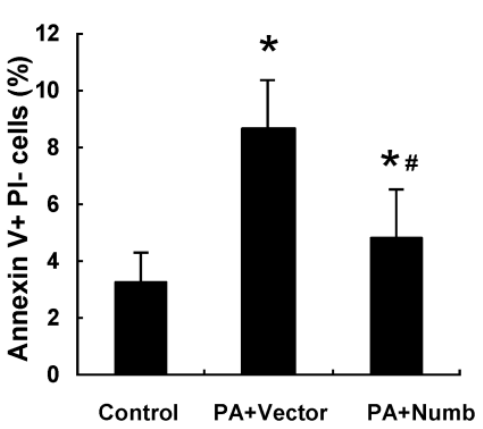

Figure 4. Overexpression of Numb attenuates PA-induced RPTC apoptosis. (A) The protein level of Numb in pAd-HA-Numb or empty vector infected cells was analyzed by Western blot. (B) NRK52E cells were infected with pAd-HA-Numb or empty vector $24 \mathrm{~h}$ before PA treatment $(100 \mu \mathrm{g} / \mathrm{ml}$ for $24 \mathrm{~h})$. Western blot was performed to analyze the expression of Numb, p53, Hes-I and cleaved caspase-3. GAPDH was used to verify equivalent loading. (C) Graphic representation of relative abundance of cleaved caspase-3 and p53 normalized to GAPDH. (D) Quantitative analysis of apoptotic NRK52E cells by flow cytometry. Each graph represents results from at least three independent experiments, and each data represents mean \pm SD from three independent experiments. $* P<0.05$ versus control without PA treatment. ${ }^{\#} P<0.05$ versus $P A$ treated empty vector infected cells. 
Numb protects RPTC from PA-induced apoptosis by inhibiting Notch signaling activity

Numb was originally identified as an intrinsic cell fate determinant in Drosophila by antagonizing Notch signaling activity. Therefore, we hypothesized that the effect of Numb on PA-induced RPTC apoptosis might be mediated by Notch signaling. To ad-

A

Figure 5. PA treatment resulted in enhanced Notch activity in a time- and dose-dependent manner. (A) NRK52E cells were treated with indicated amount of PA for $24 \mathrm{~h}$. The protein level of NICD and Hes-I was analyzed by Western blot. GAPDH was used to verify equivalent loading. (B) Graphic representation of relative abundance of NICD or Hes-I normalized to GAPDH. (C) NRK52E cells were treated with 100 $\mu \mathrm{g} / \mathrm{ml}$ of PA for the indicated time period. NICD and Hes-I protein level was analyzed by Western blot. GAPDH was used to verify equivalent loading. (D) Graphic representation of relative abundance of NICD or Hes-I normalized to GAPDH. Each graph represents results from at least three independent experiments, Data are given as means \pm SD from three independent experiments. $* P<0.05$ versus control without PA treatment.

B dress this issue, we first examine the effect of PA on the expression of the intracellular domain of Notch 1 (NICD) and Hes-1, the downstream target gene of Notch signaling. As shown in Figure 5, PA treatment significantly increased the NICD and Hes-1 in a timeand dose-dependent manner, suggesting that PA could activate Notch signaling.

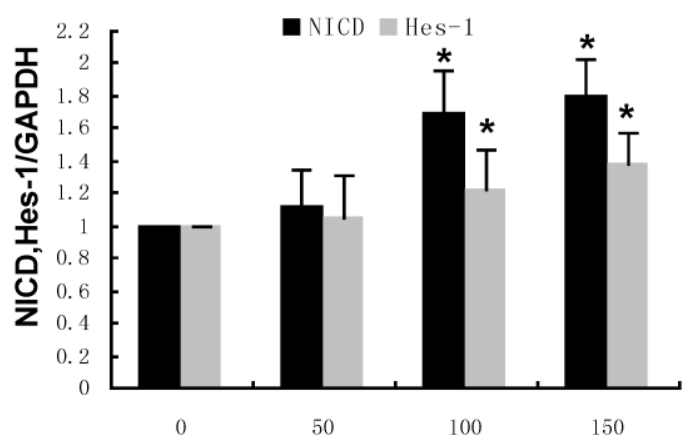

C

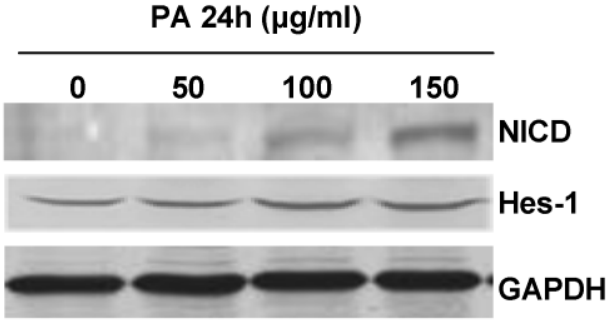

PA $100 \mu \mathrm{g} / \mathrm{ml}$ (h)

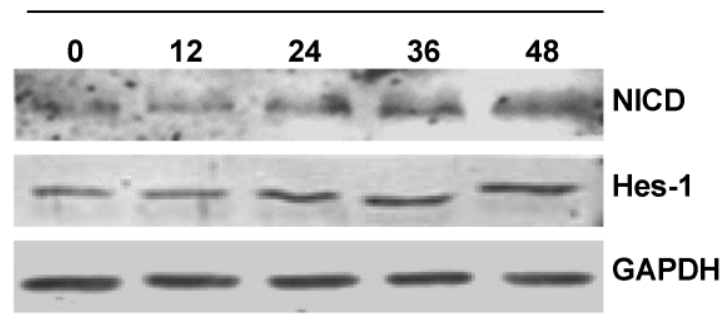

D

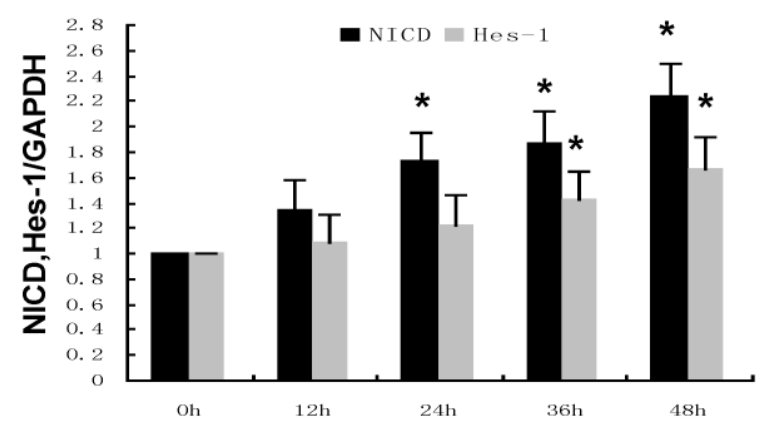


We further inhibited Notch signaling pathway using $\gamma$-secretase inhibitor DAPT in Numb-siRNA transfected cells. The efficiency of DAPT on inhibiting Notch signaling was demonstrated by decreased protein level of Hes-1(Figure 6A). As shown in Figure 6A-C, DAPT treatment led to $40 \%$ decrease of Numb-siRNA-augmented apoptosis compared to vehicle control. We next examined whether activation of Notch signaling could reverse the protective effects of Numb overexpression on PA-induced apoptosis.

A

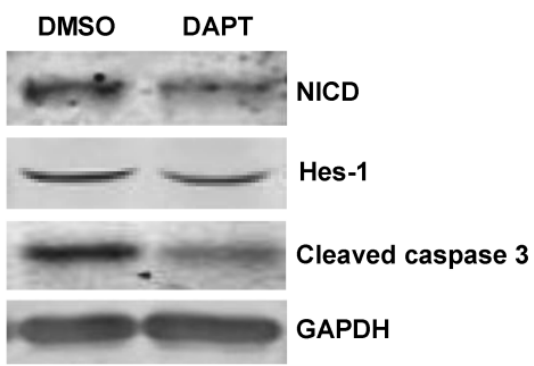

B

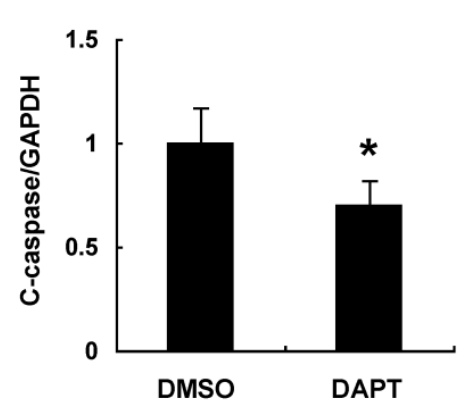

C

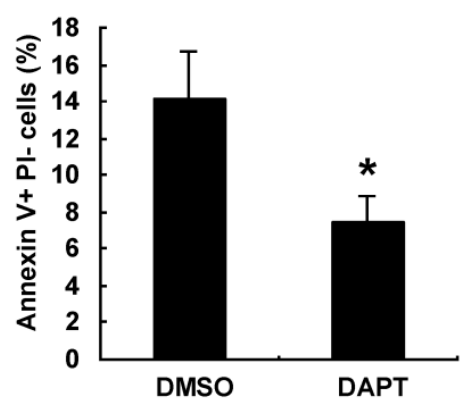

To address this issue, we overexpressed NICD by transfecting pCDNA-NICD before pAd-HA-Numb infection. As shown in Fig. 6D-F, NICD overexpression resulted in enhanced expression of Hes-1 and $20 \%$ increase of apoptosis after PA treatment compared to vector control cells. Taken together, these data provided evidence that Numb protects RPTC from PA-induced apoptosis through inhibiting Notch signaling activity.

D

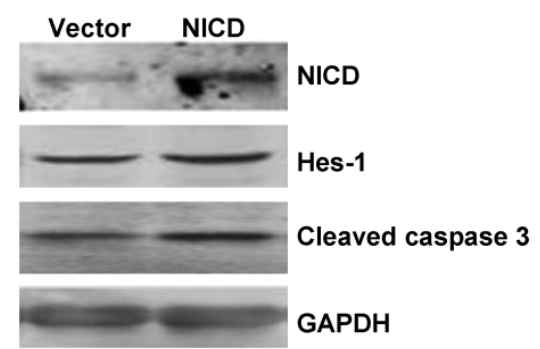

E

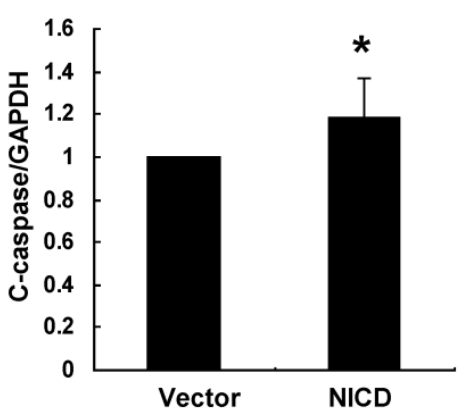

$\mathbf{F}$

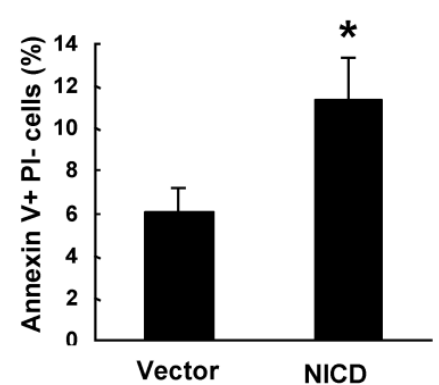

Figure 6. The role of Numb on PA-induced RPTC apoptosis is by antagonizing Notch signaling. (A) NRK52E cells were incubated with Notch signaling inhibitor DAPT or vehicle control (DMSO) $24 \mathrm{~h}$ after Numb siRNA transfection, and then NRK52E cells were treated with $100 \mu \mathrm{g} / \mathrm{ml}$ of PA for $24 \mathrm{~h}$. Western blot analysis of the protein expression of NICD, Hes- I and cleaved caspase-3. GAPDH was used to verify equivalent loading. (B) Graphic representation of relative abundance of cleaved caspase-3 normalized to GAPDH. (C) Quantitative analysis of apoptotic NRK52E cells by flow cytometry. Data are given as mean \pm SD from three independent experiments, $* P<0.05$ versus DMSO treated cells. (D) NRK52E cells were transfected with PCDNA-NICD or empty vector $24 \mathrm{~h}$ after pAd-HA-Numb infection, and then NRK52E cells were treated with $100 \mu \mathrm{g} / \mathrm{ml}$ of PA for $24 \mathrm{~h}$. Western blot analysis of the protein expression of NICD, Hes-I and cleaved caspase-3. GAPDH was used to verify equivalent loading. (E) Graphic representation of relative abundance of cleaved caspase-3 normalized to GAPDH. (F) Quantitative analysis of apoptotic NRK52E cells by flow cytometry. Data are given as mean \pm SD from three independent experiments. $* P<0.05$ versus vector transfected cells. 


\section{Discussion}

Many studies have shown that cell injury induced by PA was solely mediated by oxidative stress $[21,22]$. Suzuki showed that PA induced overproduction of ROS and apoptosis of podocytes, in vitro, using podocyte sieving from glomeruli [23]. Moreover, Sanwal et al demonstrated that the administration of ROS scavengers such as SOD and DMTU could suppress apoptosis induced by PA [24]. Consistently, Oba et al demonstrated that the administration of antioxidants could ameliorate PA-induced apoptosis [25]. To investigate the mechanism underlying oxidative stress induced apoptosis, we first investigated Numb expression in response to PA treatment and demonstrated that exposure of RPTC to PA downregulated Numb expression in a time- and dose-dependent manner. Reduction of Numb expression with siRNA potentiated PA-induced apoptotic cell death. Conversely, overexpression of Numb attenuated PA-induced apoptosis. Taken together, we characterized a novel function of Numb as a survival factor in RPTC and its upregulation protects RPTC from apoptosis caused by oxidative stress.

Caspase-3 is an effector caspase that is involved in many forms of apoptosis [26]. However, caspase-3 is still dispensable for certain forms of apoptosis [27]. Previous studies have shown that PA caused caspase-3 activation in podocyte apoptosis [23]. Consistent with previous study, our results showed that PA increased the activity of caspase-3, suggesting that PA-induced apoptosis in RPTC is caspase-3 dependent.

Previous study has shown that Numb could prevent p53 degradation in human mammary cells and thus promotes cell apoptosis [20]. In line with previous study, the protein level of p53 in Numb siRNA transfected RPTC was dramatically decreased, whereas, overexpression of Numb led to a significant increase of p53 expression. However, increased p53 did not prevent Numb-augmented RPTC apoptosis induced by PA, and vice versa. These data suggest that the role of Numb in PA-induced RPTC apoptosis is p53 independent, which is different from that in mammary epithelial cells. In addition, we noticed that the protein level of p53 in RPTC was decreased following PA exposure, which is different from that of PA-induced podocyte apoptosis. The different effect of PA on apoptosis might be due to the tissue-specific expression of Numb since we were not able to detect Numb expression in podocyte, whereas, Numb was abundant in proximal tubules (data not shown).

Numb was originally identified as an intrinsic cell fate determinant in Drosophila by antagonizing Notch signaling [13-15]. In mammals, it has been demonstrated that Numb interacts with Notch through its NICD domain and domain and promotes NICD ubiquitination and degradation [28, 29]. Given the critical role of Notch signaling in modulating the balance between cell proliferation, differentiation, and apoptosis that affect the development and function of many organs $[30,31]$, we hypothesized that Numb regulates PA-induced RPTC apoptosis through regulating the activity of Notch signaling. To address this issue, we first treated RPTC with Notch signaling inhibitor $Y$-secretase inhibitor-DAPT prior Numb-siRNA transfection and found that DAPT could reverse Numb-siRNA augmented RPTC apoptosis induced by PA. On the other hand, the protective effect of Numb overexpression on PA-induced apoptosis in RPTC was inhibited by NICD overexpression. These results indicated that Numb modulates oxidative stress-induced RPTC apoptosis through antagonizing Notch signaling activity. It has become increasingly clear that the biological function of Notch signaling is highly context-dependent. In human acute T-lymphoblastic leukemia and lymphomas, dysfunction of intracellular Notch prevents differentiation and predisposes undifferentiated cells to malignant transformation [32]. Conversely, constitutive activation of Notch signaling leads to profound growth arrest in small cell lung cancer cells, prostate cancer cells and keratinocytes [33-35]. Our data is consistent with previous findings that active Notch signaling promotes cell apoptosis.

In summary, in the present study, we demonstrated that PA induced RPTC apoptosis in a caspase-3 dependent manner. Knocking down endogenous Numb promoted PA-induced apoptosis, whereas, overexpressing Numb attenuated PA-induced apoptosis. Our study, for the first time, provides evidence that Numb has a protective effect on oxidative-induced apoptosis of RPTC and the effect of Numb on PA-induced apoptosis is through antagonizing Notch signaling activity. There is a growing body of evidence that oxidative stress plays a critical role not only in AKI but also in the progression to end stage renal failure. Accumulating evidence have demonstrated that hypertension, dyslipidaemia, diabetes, and obesity cause renal failure through overproduction of oxidative stress. Vasoactive substances such as angiotensin II and aldosterone also produce oxidative stress, resulting in renal damage [25]. Therefore, this study provides a new insight for the pathophysiology of renal failure. 


\section{Acknowledgement}

This work was supported by the National Natural Science Foundation of China (30871033 and 81070554).

\section{Conflict of Interests}

The authors have declared that no conflict of interest exists.

\section{References}

1. Bengatta S, Arnould C, Letavernier E, et al. MMP9 and SCF protect from apoptosis in acute kidney injury. J Am Soc Nephrol. 2009; 20: 787-797.

2. Daemen MA, van 't Veer C, Denecker G, et al. Inhibition of apoptosis induced by ischemia-reperfusion prevents inflammation. J Clin Invest. 1999; 104: 541-549.

3. Daemen MA, de Vries B, Buurman WA. Apoptosis and inflammation in renal reperfusion injury. Transplantation. 2002; 73: 1693-1700.

4. Hamar P, Song E, Kokeny G, et al. Small interfering RNA targeting Fas protects mice against renal ischemia-reperfusion injury. Proc Natl Acad Sci U S A. 2004; 101: 14883-14888.

5. Lorz C, Ortiz A, Justo P, et al. Proapoptotic Fas ligand is expressed by normal kidney tubular epithelium and injured glomeruli. J Am Soc Nephrol. 2000; 11: 1266-1277.

6. Saikumar P, Venkatachalam MA. Role of apoptosis in hypoxic/ischemic damage in the kidney. Semin Nephrol. 2003; 23: 511-521.

7. Kaushal GP, Singh AB, Shah SV. Identification of gene family of caspases in rat kidney and altered expression in ischemia-reperfusion injury. Am J Physiol. 1998; 274: F587-595.

8. Lameire N. The pathophysiology of acute renal failure. Crit Care Clin. 2005; 21: 197-210.

9. Lameire N, Van Biesen W, Vanholder R. Acute renal failure. Lancet. 2005; 365: 417-430.

10. Avunduk MC, Yurdakul T, Erdemli E, et al. Prevention of renal damage by alpha tocopherol in ischemia and reperfusion models of rats. Urol Res. 2003; 31: 280-285.

11. Yard BA, Daha MR, Kooymans-Couthino M, et al. IL-1 alpha stimulated TNF alpha production by cultured human proximal tubular epithelial cells. Kidney Int. 1992; 42: 383-389.

12. Yan B, Omar FM, Das K, et al. Characterization of Numb expression in astrocytomas. Neuropathology. 2008; 28: 479-484.

13. Spana EP, Doe CQ. Numb antagonizes Notch signaling to specify sibling neuron cell fates. Neuron. 1996; 17: 21-26.

14. Zhong W, Feder JN, Jiang MM, et al. Asymmetric localization of a mammalian numb homolog during mouse cortical neurogenesis. Neuron. 1996; 17: 43-53.

15. Guo M, Jan LY, Jan YN. Control of daughter cell fates during asymmetric division: interaction of Numb and Notch. Neuron. 1996; 17: 27-41.

16. Verdi JM, Schmandt R, Bashirullah A, et al. Mammalian NUMB is an evolutionarily conserved signaling adapter protein that specifies cell fate. Curr Biol. 1996; 6: 1134-1145.

17. Verdi JM, Bashirullah A, Goldhawk DE, et al. Distinct human NUMB isoforms regulate differentiation vs. proliferation in the neuronal lineage. Proc Natl Acad Sci U S A. 1999; 96: 10472-10476.

18. Dho SE, French MB, Woods SA, et al. Characterization of four mammalian numb protein isoforms. Identification of cytoplasmic and membrane-associated variants of the phosphotyrosine binding domain. J Biol Chem. 1999; 274: 33097-33104.

19. Pedersen WA, Chan SL, Zhu H, et al. Numb isoforms containing a short PTB domain promote neurotrophic factor-induced differentiation and neurotrophic factor withdrawal-induced death of PC12 Cells. J Neurochem. 2002; 82: 976-986.

20. Colaluca IN, Tosoni D, Nuciforo P, et al. NUMB controls p53 tumour suppressor activity. Nature. 2008; 451: 76-80.

21. Someya T, Kaneko K, Yamada T, et al. Effect of a novel free radical scavenger, edaravone, on puromycin aminonucleoside induced nephrosis in rats. Pediatr Nephrol. 2005; 20: 1430-1434.

22. Rincon J, Romero M, Viera N, et al. Increased oxidative stress and apoptosis in acute puromycin aminonucleoside nephrosis. Int J Exp Pathol. 2004; 85: 25-33.

23. Suzuki T, Takemura H, Noiri E, et al. Puromycin aminonucleoside induces apoptosis and increases HNE in cultured glomerular epithelial cells(1). Free Radic Biol Med. 2001; 31: 615-623.

24. Sanwal V, Pandya M, Bhaskaran M, et al. Puromycin aminonucleoside induces glomerular epithelial cell apoptosis. Exp Mol Pathol. 2001; 70: 54-64.

25. Oba S, Hino M, Fujita T. Adrenomedullin protects against oxidative stress-induced podocyte injury as an endogenous antioxidant. Nephrol Dial Transplant. 2008; 23: 510-517.

26. Nunez G, Benedict MA, Hu Y, et al. Caspases: the proteases of the apoptotic pathway. Oncogene. 1998; 17: 3237-3245.

27. Mohr S, McCormick TS, Lapetina EG. Macrophages resistant to endogenously generated nitric oxide-mediated apoptosis are hypersensitive to exogenously added nitric oxide donors: dichotomous apoptotic response independent of caspase 3 and reversal by the mitogen-activated protein kinase kinase (MEK) inhibitor PD 098059. Proc Natl Acad Sci U S A. 1998; 95: 5045-5050.

28. Wakamatsu Y, Maynard TM, Jones SU, et al. NUMB localizes in the basal cortex of mitotic avian neuroepithelial cells and modulates neuronal differentiation by binding to NOTCH-1. Neuron. 1999; 23(1): 71-81.

29. McGill MA, McGlade CJ. Mammalian numb proteins promote Notch1 receptor ubiquitination and degradation of the Notch1 intracellular domain. J Biol Chem. 2003; 278(25): 23196-203.

30. Ohishi K, Katayama N, Shiku H, et al. Notch signaling in hematopoiesis. Semin Cell Dev Biol. 2003; 14: 143-150.

31. Weijzen S, Rizzo P, Braid M, et al. Activation of Notch-1 signaling maintains the neoplastic phenotype in human Ras-transformed cells. Nat Med. 2002; 8: 979-986.

32. Pear WS, Aster JC, Scott ML, et al. Exclusive development of T cell neoplasms in mice transplanted with bone marrow expressing activated Notch alleles. J Exp Med. 1996; 183: 2283-2291.

33. Sriuranpong V, Borges MW, Ravi RK, et al. Notch signaling induces cell cycle arrest in small cell lung cancer cells. Cancer Res. 2001; 61: 3200-3205.

34. Shou J, Ross S, Koeppen H, et al. Dynamics of notch expression during murine prostate development and tumorigenesis. Cancer Res. 2001; 61: 7291-7297.

35. Rangarajan A, Talora C, Okuyama R, et al. Notch signaling is a direct determinant of keratinocyte growth arrest and entry into differentiation. Embo J. 2001; 20: 3427-3436. 